\title{
PERMUTATION TEST FOR EQUALITY OF EACH CHARACTERISTIC ROOT IN TWO POPULATIONS
}

\author{
Yuichi Takeda*
}

\begin{abstract}
We consider the problem of testing the equality of intermediate characteristic roots in two populations. The permutation test is investigated for testing the hypothesis. The exact distribution of the ratio of the largest characteristic roots across populations is derived under the assumption of multivariate normality. A Monte Carlo experiment is conducted to examine the performance of the permutation test under the assumptions that two population distributions are characterized by multivariate normal and contaminated multivariate normal distributions.
\end{abstract}

\section{Introduction}

Principal component analysis is a multivariate technique to reduce the dimension of multivariate data, and algebraically based on characteristic roots and vectors of a sample covariance matrix. Suppose that $\boldsymbol{X}^{(1)}$ and $\boldsymbol{X}^{(2)}$ are $p$-variate random vectors with mean vectors $\boldsymbol{\mu}^{(i)}$ and covariance matrices $\boldsymbol{\Sigma}^{(i)}, i=1,2$. Let $\lambda_{1}^{(i)} \geq \cdots \geq \lambda_{p}^{(i)}$ be the ordered characteristic roots of $\boldsymbol{\Sigma}^{(i)}$, and let $\boldsymbol{\beta}_{1}^{(i)}, \cdots, \boldsymbol{\beta}_{p}^{(i)}$ the corresponding normalized characteristic vectors. We are interested in testing the hypothesis

$$
H_{0}: \lambda_{j}^{(1)}=\lambda_{j}^{(2)}
$$

for $j=1, \cdots, p$. For example, the variables $\boldsymbol{X}^{(1)}$ and $\boldsymbol{X}^{(2)}$ are the data of the test on the same subject given to the second and third grade students of a junior high school. In this case, it is of interest to test the hypothesis for the largest characteristic roots. And we consider the case where the $\boldsymbol{X}^{(1)}$ and $\boldsymbol{X}^{(2)}$ are the data of Japanese and European clothes observed from the same items, and some other cases, besides (Sugiyama, 1983). In this case, we do not know that the population distribution is the multivariate normal distribution or not. Thus we may consider non-parametric methods.

In this paper, we consider the permutation test for testing the hypothesis $H_{0}$. Sugiyama and Ushizawa (1998) gave the Ansari-Bradley test for the above testing when independent samples are drawn from multivariate non-normal populations and some simulation results. In Section 2, we give the permutation test of equality of characteristic roots. For comparison of the power of the permutation test, we derive the exact distribution of the test statistic given as the ratio of two characteristic roots of sample covariance matrices in Section 3 . In Section 4, we examine the performance of the permutation test, using a Monte Carlo simulation, when two population distributions are characterized by the multivariate normal and contaminated multivariate normal distributions. The discussions of a simulation study are summarized in Section 5.

* Department of Mathematics, Chuo University, 1-13-27, Kasuga, Bunkyo-ku, Tokyo 112-8551, Japan

Key words: Principal component; Two sample problem; Exact distribution; Powers 


\section{Permutation test of equality of characteristic roots}

Suppose that $\boldsymbol{x}_{1}^{(1)}, \cdots, \boldsymbol{x}_{N_{1}}^{(1)}$ and $\boldsymbol{x}_{1}^{(2)}, \cdots, \boldsymbol{x}_{N_{2}}^{(2)}$ be independent samples from two $p$ variate distributions. We consider the following null hypothesis for the fixed $j$;

$$
H_{0}: \lambda_{j}^{(1)}=\lambda_{j}^{(2)} \text {. }
$$

The null hypothesis is true if and only if $\operatorname{Var}\left[\boldsymbol{\beta}_{j}^{(1)^{\prime}} \boldsymbol{X}^{(1)}\right]$ and $\operatorname{Var}\left[\boldsymbol{\beta}_{j}^{(2)^{\prime}} \boldsymbol{X}^{(2)}\right]$ are equal. We consider the permutation test for equality of variance $\boldsymbol{\beta}_{j}^{(i)^{\prime}} \boldsymbol{X}^{(i)}$ for $i=1,2$ (Good, 1994). Let

$$
y_{j k}^{(i)}=\boldsymbol{\beta}_{j}^{(i)^{\prime}}\left(\boldsymbol{x}_{k}^{(i)}-\boldsymbol{\mu}^{(i)}\right), \quad k=1,2, \cdots, N_{i}, i=1,2 .
$$

If the distributions of the variables $y_{j k}^{(i)}$ for $i=1,2$ are the same, the variables $Y=$ $\left\{y_{j 1}^{(1)}, \cdots, y_{j N_{1}}^{(1)}, y_{j 1}^{(2)}, \cdots, y_{j N_{2}}^{(2)}\right\}$ are exchangeable (see Pesarin (1999)). Hence we consider the permutation of $y_{j k}^{(i)}$ and the test of equality of each variance under the null hypothesis, based on the following test statistic

$$
\sum_{k_{2}=1}^{N_{2}} y_{j k_{2}}^{(2)^{2}} / \sum_{k_{1}=1}^{N_{1}} y_{j k_{1}}^{(1)^{2}} .
$$

The expected value of $\sum_{k=1}^{N_{i}} y_{j k}^{(i)^{2}}$ is $\lambda_{j}^{(i)}$. If the null hypothesis is true, the test statistic should be close to 1 .

Let $\ell_{1}^{(i)} \geq \cdots \geq \ell_{p}^{(i)}$ be the characteristic roots of the sample covariance matrix $\boldsymbol{S}^{(i)}$ given by $(5)$, and $\boldsymbol{b}_{1}^{(\bar{i})}, \cdots, \boldsymbol{b}_{p}^{(i)}$ be the corresponding normalized characteristic vectors. Then corresponding to $(1)$ replace

$$
z_{j k}^{(i)}=\boldsymbol{b}_{j}^{(i)^{\prime}}\left(\boldsymbol{x}_{k}^{(i)}-\overline{\boldsymbol{x}}^{(i)}\right), \quad k=1,2, \cdots, N_{i}, i=1,2
$$

We may assume that $Z=\left\{z_{j 1}^{(1)}, \cdots, z_{j N_{1}}^{(1)}, z_{j 1}^{(2)}, \cdots, z_{j N_{2}}^{(2)}\right\}$ are approximately exchangeable for large sample size. Thus we apply the permutation test for equality of variances between $z_{j k}^{(1)}$ and $z_{j k}^{(2)}$. Instead of (2), we consider the following statistic

$$
\begin{aligned}
T_{Z} & =\sum_{k_{2}=1}^{N_{2}} z_{j k_{2}}^{(2)^{2}} / \sum_{k_{1}=1}^{N_{1}} z_{j k_{1}}^{(1)^{2}} \\
& =\ell_{j}^{(2)} / \ell_{j}^{(1)} .
\end{aligned}
$$

The $Z^{*}$ is a permutation data of the $Z$, and we calculate the statistic $T_{Z^{*}}$ based on $Z^{*}$. For large $N_{i}$, it is difficult to obtain all permutation data. In Section 4 we permute just 10,000 times, and estimate the p-value of the test statistic $T_{Z}$.

\section{Distribution of ratio of two characteristic roots}

For comparison of the power, it is important to know the distribution of the test statistics $T$. In this section, we consider the distribution of the test statistics under the normal distribution, which is derivable. Investigation under the normal distribution is important since it is likely to provide a basis for propriety of the conjecture under different distributions. Therefore we may discuss the comparison with the exact power to examine the value 
of our method in section 5. The asymptotic distribution of ratio of two characteristic roots may be obtained by using Sugiura's result (1973). In the others, since $\log \ell_{j}^{(i)}-\log \lambda_{j}^{(i)}$ is asymptotically distributed as $N\left(0,2 / n_{i}\right)$ (see Konishi (1977)), the asymptotic distribution of $\log \ell_{j}^{(1)}-\log \ell_{j}^{(2)}$ is $N\left(0,2\left(1 / n_{1}+1 / n_{2}\right)\right)$. Hence if two populations are independently distributed with multivariate normal distribution, we may use this statistic which does not depend on population parameters under the null hypothesis.

In this section, we give the exact distribution of the test statistic $\ell_{1}^{(2)} / \ell_{1}^{(1)}$, the ratio of two largest characteristic roots, under the multivariate normal distribution for comparison of the power of permutation test.

Let each sample covariance matrix be

$$
\boldsymbol{S}^{(i)}=\frac{1}{N_{i}-1} \sum_{j=1}^{N_{i}}\left(\boldsymbol{x}_{j}^{(i)}-\overline{\boldsymbol{x}}^{(i)}\right)\left(\boldsymbol{x}_{j}^{(i)}-\overline{\boldsymbol{x}}^{(i)}\right)^{\prime} .
$$

Then $n_{i} \boldsymbol{S}^{(i)}$ has Wishart distribution

$$
W_{p}\left(\boldsymbol{S}^{(i)} ; n_{i}, n_{i}^{-1} \boldsymbol{\Sigma}^{(i)}\right)=\frac{\left|\boldsymbol{S}^{(i)}\right|^{\frac{1}{2}\left(n_{i}-p-1\right)} \exp \left\{-\frac{1}{2} n_{i} \operatorname{tr} \boldsymbol{\Sigma}^{(i)^{-1}} \boldsymbol{S}^{(i)}\right\}}{2^{\frac{1}{2} p n_{i}} \pi^{\frac{1}{4} p(p-1)}\left|\frac{1}{n_{i}} \boldsymbol{\Sigma}^{(i)}\right|^{\frac{1}{2} n_{i}} \prod_{j=1}^{p} \Gamma\left[\frac{1}{2}\left(n_{i}+1-j\right)\right]},
$$

where $n_{i}=N_{i}-1$. Sugiyama (1967) gave the following cumulative distribution function of the largest characteristic root $\ell_{1}$ of the Wishart matrix $W_{p}\left(\boldsymbol{S}, n, n^{-1} \boldsymbol{\Sigma}\right)$

$$
\begin{aligned}
P\left\{\ell_{1}<x\right\}= & C(\boldsymbol{\Sigma}, n) \exp \left(-\frac{n x}{2} \operatorname{tr} \boldsymbol{\Sigma}^{-1}\right)(n x)^{\frac{1}{2} p n} \\
& { }_{1} F_{1}\left(\frac{p+1}{2} ; \frac{n+p+1}{2} ; \frac{n x}{2} \boldsymbol{\Sigma}^{-1}\right),
\end{aligned}
$$

where

$$
\begin{aligned}
C(\boldsymbol{\Sigma}, n) & =|\boldsymbol{\Sigma}|^{-\frac{1}{2} n} \Gamma_{p}\left(\frac{p+1}{2}\right) / 2^{\frac{1}{2} n p} \Gamma_{p}\left(\frac{n+p+1}{2}\right) \\
\Gamma_{p}(n) & =\pi^{\frac{p(p-1)}{4}} \prod_{i=1}^{p} \Gamma\left(n-\frac{1}{2}(i-1)\right)
\end{aligned}
$$

and $n=N-1$. Let $\tilde{n}=p n / 2$ and $\tilde{\boldsymbol{\Sigma}}^{-1}=\frac{1}{2} \boldsymbol{\Sigma}^{-1}$, then we rewrite the hypergeometric function ${ }_{1} F_{1}(a ; c ; Y)$ in terms of infinite series as

$$
\begin{gathered}
P\left\{\ell_{1}<x\right\}=C(\boldsymbol{\Sigma}, n) n^{\frac{1}{2} p n} \sum_{k=0}^{\infty} \sum_{\kappa} \frac{\left(\frac{p+1}{2}\right)_{\kappa}}{\left(\frac{n+p+1}{2}\right)_{\kappa}} \frac{C_{\kappa}\left(n \tilde{\boldsymbol{\Sigma}}^{-1}\right)}{k !} \\
\cdot x^{\tilde{n}+k} \exp \left(-x \operatorname{tr} n \tilde{\boldsymbol{\Sigma}}^{-1}\right)
\end{gathered}
$$

where

$$
\begin{aligned}
(\alpha)_{\kappa} & =\prod_{i=1}^{p}\left(\alpha-\frac{1}{2}(i-1)\right)_{k_{i}}, \\
(\alpha)_{k_{i}} & =\alpha(\alpha+1) \cdots\left(\alpha+k_{i}-1\right), \quad(\alpha)_{0}=1 .
\end{aligned}
$$




\section{TAKEDA}

The function $C_{\kappa}(Y)$ is a zonal polynomial of the symmetric matrix $Y$ corresponding to the partition $\kappa=\left(k_{1}, k_{2}, \cdots, k_{p}\right)$, with $\sum_{i} k_{i}=k$ and $k_{1} \geq k_{2} \geq \cdots \geq k_{p} \geq 0$. Taking the derivative of (8) with respect to $x$, we obtain the following probability density function

$$
\begin{aligned}
f(x)= & C(\boldsymbol{\Sigma}, n) n^{\frac{1}{2} p n} \sum_{k=0}^{\infty} \sum_{\kappa} \frac{\left(\frac{p+1}{2}\right)_{\kappa}}{\left(\frac{n+p+1}{2}\right)_{\kappa}} \frac{C_{\kappa}\left(n \tilde{\boldsymbol{\Sigma}}^{-1}\right)}{k !} \\
& \cdot\left\{(\tilde{n}+k) x^{\tilde{n}+k-1}-\left(\operatorname{tr} n \tilde{\boldsymbol{\Sigma}}^{-1}\right) x^{\tilde{n}+k}\right\} \\
& \cdot \exp \left(-x \operatorname{tr} n \tilde{\boldsymbol{\Sigma}}^{-1}\right) .
\end{aligned}
$$

Hence, we have the joint distribution $g(x, y)$ of two largest characteristic roots $\ell_{1}^{(1)}$ and $\ell_{1}^{(2)}$ from (9) as follows

$$
\begin{aligned}
g(x, y)= & C\left(\boldsymbol{\Sigma}^{(1)}, n_{1}\right) C\left(\boldsymbol{\Sigma}^{(2)}, n_{2}\right) n_{1}^{\tilde{n}_{1}} n_{2}^{\tilde{n}_{2}} \\
& \cdot \sum_{k=0}^{\infty} \sum_{k^{\prime}=0}^{\infty} \sum_{\kappa} \sum_{\kappa^{\prime}} \frac{\left(\frac{p+1}{2}\right)_{\kappa}}{\left(\frac{n_{1}+p+1}{2}\right)_{\kappa}} \frac{\left(\frac{p+1}{2}\right)_{\kappa^{\prime}}}{\left(\frac{n_{2}+p+1}{2}\right)_{\kappa^{\prime}}} \frac{C_{\kappa}\left(n_{1} \tilde{\boldsymbol{\Sigma}}_{1}^{-1}\right)}{k !} \frac{C_{\kappa^{\prime}}\left(n_{2} \tilde{\boldsymbol{\Sigma}}_{2}^{-1}\right)}{k^{\prime} !} \\
& \cdot\left\{\left(\tilde{n}_{1}+k\right) x^{\tilde{n}_{1}+k-1}-\left(\operatorname{tr} n_{1} \tilde{\boldsymbol{\Sigma}}_{1}^{-1}\right) x^{\tilde{n}_{1}+k}\right\} \\
& \cdot\left\{\left(\tilde{n}_{2}+k^{\prime}\right) y^{\tilde{n}_{2}+k^{\prime}-1}-\left(\operatorname{tr} n_{2} \tilde{\boldsymbol{\Sigma}}_{2}^{-1}\right) y^{\tilde{n}_{2}+k^{\prime}}\right\} \\
& \cdot \exp \left(-x \operatorname{tr} n_{1} \tilde{\boldsymbol{\Sigma}}_{1}^{-1}\right) \exp \left(-y \operatorname{tr} n_{2} \tilde{\boldsymbol{\Sigma}}_{2}^{-1}\right),
\end{aligned}
$$

where

$$
\tilde{n}_{i}=\frac{1}{2} p n_{i}, \tilde{\mathbf{\Sigma}}_{i}^{-1}=\frac{1}{2} \Sigma^{(i)^{-1}}
$$

To derive the marginal distribution of the ratio test statistic (4), we transform $x$ and $y$ to $u$ and $v$

$$
u=\frac{y}{x}, v=x \quad\{u>0, v>0\} .
$$

Then we have the following joint distribution of the variable $u$ and $v$

$$
\begin{aligned}
g(u, v)= & C\left(\boldsymbol{\Sigma}^{(1)}, n_{1}\right) C\left(\boldsymbol{\Sigma}^{(2)}, n_{2}\right) n_{1}^{\tilde{n}_{1}} n_{2}^{\tilde{n}_{2}} \\
& \cdot \sum_{k=0}^{\infty} \sum_{k^{\prime}=0}^{\infty} \sum_{\kappa} \sum_{\kappa^{\prime}} \frac{\left(\frac{p+1}{2}\right)_{\kappa}}{\left(\frac{n_{1}+p+1}{2}\right)_{\kappa}} \frac{\left(\frac{p+1}{2}\right)_{\kappa^{\prime}}}{\left(\frac{n_{2}+p+1}{2}\right)_{\kappa^{\prime}}} \frac{C_{\kappa}\left(n_{1} \tilde{\boldsymbol{\Sigma}}_{1}^{-1}\right)}{k !} \frac{C_{\kappa^{\prime}}\left(n_{2} \tilde{\boldsymbol{\Sigma}}_{2}^{-1}\right)}{k^{\prime} !} \\
& \cdot\left\{\left(\tilde{n}_{1}+k\right)\left(\tilde{n}_{2}+k^{\prime}\right) u^{\tilde{n}_{2}+k^{\prime}-1} v^{\tilde{n}_{1}+\tilde{n}_{2}+k+k^{\prime}-1}\right. \\
& -\left(\operatorname{tr} n_{1} \tilde{\boldsymbol{\Sigma}}_{1}^{-1}\right)\left(\tilde{n}_{2}+k^{\prime}\right) u^{\tilde{n}_{2}+k^{\prime}-1} v^{\tilde{n}_{1}+\tilde{n}_{2}+k+k^{\prime}} \\
& -\left(\tilde{n}_{1}+k\right)\left(\operatorname{tr} n_{2} \tilde{\boldsymbol{\Sigma}}_{2}^{-1}\right) u^{\tilde{n}_{2}+k^{\prime}} v^{\tilde{n}_{1}+\tilde{n}_{2}+k+k^{\prime}} \\
& \left.+\left(\operatorname{tr} n_{1} \tilde{\boldsymbol{\Sigma}}_{1}^{-1}\right)\left(\operatorname{tr} n_{2} \tilde{\boldsymbol{\Sigma}}_{2}^{-1}\right) u^{\tilde{n}_{2}+k^{\prime}} v^{\tilde{n}_{1}+\tilde{n}_{2}+k+k^{\prime}+1}\right\} \\
& \cdot \exp \left\{-\left(\operatorname{tr} n_{1} \tilde{\boldsymbol{\Sigma}}_{1}+u \operatorname{tr} n_{2} \tilde{\boldsymbol{\Sigma}}_{2}\right) v\right\} .
\end{aligned}
$$


Integrating with the variable $v$ in formula (11), we obtain the distribution of the ratio $u$ of two largest characteristic roots as follows

$$
\begin{aligned}
h(u)= & C\left(\boldsymbol{\Sigma}^{(1)}, n_{1}\right) C\left(\boldsymbol{\Sigma}^{(2)}, n_{2}\right) n_{1}^{\tilde{n}_{1}} n_{2}^{\tilde{n}_{2}} \\
& \sum_{k=0}^{\infty} \sum_{k^{\prime}=0}^{\infty} \sum_{\kappa} \sum_{\kappa^{\prime}} \frac{\left(\frac{p+1}{2}\right)_{\kappa}}{\left(\frac{n_{1}+p+1}{2}\right)_{\kappa}} \frac{\left(\frac{p+1}{2}\right)_{\kappa^{\prime}}}{\left(\frac{n_{2}+p+1}{2}\right)_{\kappa^{\prime}}} \frac{C_{\kappa}\left(n_{1} \tilde{\boldsymbol{\Sigma}}_{1}^{-1}\right)}{k !} \frac{C_{\kappa^{\prime}}\left(n_{2} \tilde{\boldsymbol{\Sigma}}_{2}^{-1}\right)}{k^{\prime} !} \\
& \left\{\left(\tilde{n}_{1}+k\right)\left(\tilde{n}_{2}+k^{\prime}\right) \Gamma\left(\tilde{n}_{1}+\tilde{n}_{2}+k+k^{\prime}\right) \Delta_{0}^{-1}\right. \\
& -\left(\operatorname{tr} n_{1} \tilde{\boldsymbol{\Sigma}}_{1}\right)\left(\tilde{n}_{2}+k^{\prime}\right) \Gamma\left(\tilde{n}_{1}+\tilde{n}_{2}+k+k^{\prime}+1\right) \Delta_{1}^{-1} \\
& -\left(\tilde{n}_{1}+k\right)\left(\operatorname{tr} n_{2} \tilde{\boldsymbol{\Sigma}}_{2}\right) \Gamma\left(\tilde{n}_{1}+\tilde{n}_{2}+k+k^{\prime}+1\right) \Delta_{1}^{0} \\
& \left.+\left(\operatorname{tr} n_{1} \tilde{\boldsymbol{\Sigma}}_{1}\right)\left(\operatorname{tr} n_{2} \tilde{\boldsymbol{\Sigma}}_{2}\right) \Gamma\left(\tilde{n}_{1}+\tilde{n}_{2}+k+k^{\prime}+2\right) \Delta_{2}^{0}\right\}
\end{aligned}
$$

where

$$
\Delta_{j}^{i}=\frac{u^{\tilde{n}_{2}+k^{\prime}+i}}{\left(\operatorname{tr} n_{1} \tilde{\boldsymbol{\Sigma}}_{1}^{-1}+u \operatorname{tr} n_{2} \tilde{\boldsymbol{\Sigma}}_{2}^{-1}\right)^{\tilde{n}_{1}+\tilde{n}_{2}+k+k^{\prime}+j}} .
$$

Thus, we obtain the following theorem.

Theorem Let $\boldsymbol{S}^{(1)}$ and $\boldsymbol{S}^{(2)}$ have the independent Wishart distributions $W_{p}\left(n_{1}, n_{1}^{-1} \boldsymbol{\Sigma}^{(1)}\right)$ and $W_{p}\left(n_{2}, n_{2}^{-1} \boldsymbol{\Sigma}^{(2)}\right)$ respectively. Let $\ell_{1}^{(i)}$ be the largest characteristic roots of $\boldsymbol{S}^{(i)}$. The ratio $u$ of two largest characteristic roots,

$$
\ell_{1}^{(2)} / \ell_{1}^{(1)}
$$

are distributed as the formula (12).

\section{Simulation Study}

We examine the performance of our method by simulation study under the multivariate normal distribution and the multivariate contaminated normal distribution. We use uniform random numbers based on the $M$-sequence in the simulation study. Shiraishi and Okuyama (1991) showed that the standard normal random numbers generated by the above sequence are very close to the theoretical values of the means, variance, skewness and kurtosis of the standard normal distribution, and also superior in the viewpoints of the goodness of fit.

We consider the following null hypothesis and alternative hypothesis for fixed $j$;

$$
\begin{array}{ll}
H_{0}: & \lambda_{j}^{(1)}=\lambda_{j}^{(2)} \quad \text { and } \\
H_{1}: & \lambda_{j}^{(1)}<\lambda_{j}^{(2)} .
\end{array}
$$

The method of our permutation test is given by following:

1) Transform the data set from the given data $\boldsymbol{x}_{k}^{(i)}$ to $z_{j k}^{(i)}=\boldsymbol{b}_{j}^{(i)^{\prime}}\left(\boldsymbol{x}_{k}^{(i)}-\overline{\boldsymbol{x}}\right)$.

2) Calculate the observed value $T_{o b}$ of the test statistics $T$ from data set $Z$.

3) Compose a permutation data set $Z^{*}$.

4) Calculate $T^{*}$ from data set $\boldsymbol{Z}^{*}$. 


\section{TAKEDA}

5) Independently, repeat $B$ times steps 3) and 4).

6) When $\#\left\{T^{*} \geq T_{o b}\right\} / B<\alpha$, reject the null hypothesis with $100(1-\alpha) \%$ significance level, otherwise do not reject it.

For all cases, 10,000 time permutations in each simulation were performed, and we simulated 10,000 times to obtain the actual significance level.

The purpose of these simulations is to examine the actual significance levels corresponding to the nominal levels, in the following three cases, (i) the covariance matrices are equal, (ii) the characteristic roots are equal and (iii) the largest characteristic roots are equal. First, we examine the case of normal distribution. Let $\boldsymbol{x}_{1}^{(i)}, \cdots, \boldsymbol{x}_{N_{i}}^{(i)}$ be a sample from the normal distribution $N\left(\mathbf{0}, \boldsymbol{\Sigma}^{(i)}\right)$. We consider the following cases:

Case 1. Covariance matrices are equal.

$$
\boldsymbol{\Sigma}^{(1)}=\boldsymbol{\Sigma}^{(2)}=\operatorname{diag}(6.0,3.0,1.0)
$$

Case 2. All of the characteristic roots in two populations are equal.

$$
\boldsymbol{\Sigma}^{(1)}=\operatorname{diag}(6.0,3.0,1.0), \quad \boldsymbol{\Sigma}^{(2)}=\left(\begin{array}{ccc}
5.4776 & -0.7244 & -1.1998 \\
-0.7244 & 3.2010 & 0.1941 \\
-1.1998 & 0.1941 & 1.3215
\end{array}\right)
$$

Case 3. The largest characteristic roots in two populations are equal.

$$
\boldsymbol{\Sigma}^{(1)}=\operatorname{diag}(6.0,3.0,1.0), \quad \boldsymbol{\Sigma}^{(2)}=\operatorname{diag}(6.0,4.0,2.0)
$$

Tables 1, 2 and 3 show the actual significance levels corresponding to the nominal levels.

Table 1: Actual significance levels corresponding to the nominal levels for Case 1

\begin{tabular}{ccccccc}
\hline$n_{1}=n_{2}=20$ & $2.5 \%$ & $5 \%$ & $10 \%$ & $90 \%$ & $95 \%$ & $97.5 \%$ \\
\hline$\lambda_{1}^{(1)}=\lambda_{1}^{(2)}$ & 0.0156 & 0.0355 & 0.0820 & 0.9193 & 0.9637 & 0.9845 \\
$\lambda_{2}^{(1)}=\lambda_{2}^{(2)}$ & 0.0217 & 0.0450 & 0.0958 & 0.9071 & 0.9562 & 0.9791 \\
$\lambda_{3}^{(1)}=\lambda_{3}^{(2)}$ & 0.0340 & 0.0610 & 0.1157 & 0.8853 & 0.9379 & 0.9666 \\
\hline \hline$n_{1}=n_{2}=50$ & $2.5 \%$ & $5 \%$ & $10 \%$ & $90 \%$ & $95 \%$ & $97.5 \%$ \\
\hline$\lambda_{1}^{(1)}=\lambda_{1}^{(2)}$ & 0.0213 & 0.0440 & 0.0930 & 0.9083 & 0.9567 & 0.9793 \\
$\lambda_{2}^{(1)}=\lambda_{2}^{(2)}$ & 0.0238 & 0.0483 & 0.0982 & 0.8986 & 0.9492 & 0.9763 \\
$\lambda_{3}^{(1)}=\lambda_{3}^{(2)}$ & 0.0231 & 0.0468 & 0.0968 & 0.9036 & 0.9534 & 0.9768 \\
\hline \hline$n_{1}=n_{2}=100$ & $2.5 \%$ & $5 \%$ & $10 \%$ & $90 \%$ & $95 \%$ & $97.5 \%$ \\
\hline$\lambda_{1}^{(1)}=\lambda_{1}^{(2)}$ & 0.0231 & 0.0468 & 0.0970 & 0.9036 & 0.9534 & 0.9768 \\
$\lambda_{2}^{(1)}=\lambda_{2}^{(2)}$ & 0.0232 & 0.0466 & 0.0955 & 0.8975 & 0.9482 & 0.9742 \\
$\lambda_{3}^{(1)}=\lambda_{3}^{(2)}$ & 0.0255 & 0.0507 & 0.0994 & 0.8978 & 0.9511 & 0.9751 \\
\hline
\end{tabular}


Permutation Test for Equality of Each Characteristic Root

Table 2: Actual significance levels corresponding to the nominal levels for Case 2

\begin{tabular}{ccccccc}
\hline$n_{1}=n_{2}=20$ & $2.5 \%$ & $5 \%$ & $10 \%$ & $90 \%$ & $95 \%$ & $97.5 \%$ \\
\hline$\lambda_{1}^{(1)}=\lambda_{1}^{(2)}$ & 0.0158 & 0.0331 & 0.0795 & 0.9187 & 0.9646 & 0.9845 \\
$\lambda_{2}^{(1)}=\lambda_{2}^{(2)}$ & 0.0235 & 0.0466 & 0.1006 & 0.9019 & 0.9535 & 0.9768 \\
$\lambda_{3}^{(1)}=\lambda_{3}^{(2)}$ & 0.0340 & 0.0610 & 0.1157 & 0.8853 & 0.9379 & 0.9666 \\
\hline \hline$n_{1}=n_{2}=50$ & $2.5 \%$ & $5 \%$ & $10 \%$ & $90 \%$ & $95 \%$ & $97.5 \%$ \\
\hline$\lambda_{1}^{(1)}=\lambda_{1}^{(2)}$ & 0.0215 & 0.0448 & 0.0901 & 0.9051 & 0.9548 & 0.9781 \\
$\lambda_{2}^{(1)}=\lambda_{2}^{(2)}$ & 0.0213 & 0.0473 & 0.0975 & 0.9042 & 0.9520 & 0.9756 \\
$\lambda_{3}^{(1)}=\lambda_{3}^{(2)}$ & 0.0231 & 0.0468 & 0.0968 & 0.9036 & 0.9534 & 0.9768 \\
\hline \hline$n_{1}=n_{2}=100$ & $2.5 \%$ & $5 \%$ & $10 \%$ & $90 \%$ & $95 \%$ & $97.5 \%$ \\
\hline$\lambda_{1}^{(1)}=\lambda_{1}^{(2)}$ & 0.0229 & 0.0443 & 0.0970 & 0.9044 & 0.9527 & 0.9766 \\
$\lambda_{2}^{(1)}=\lambda_{2}^{(2)}$ & 0.0223 & 0.0482 & 0.1019 & 0.9022 & 0.9520 & 0.9762 \\
$\lambda_{3}^{(1)}=\lambda_{3}^{(2)}$ & 0.0270 & 0.0521 & 0.1068 & 0.8980 & 0.9476 & 0.9747 \\
\hline
\end{tabular}

Table 3: Actual significance levels corresponding to the nominal levels for Case 3

\begin{tabular}{ccccccc}
\hline$n_{1}=n_{2}=20$ & $2.5 \%$ & $5 \%$ & $10 \%$ & $90 \%$ & $95 \%$ & $97.5 \%$ \\
\hline$\lambda_{1}^{(1)}=\lambda_{1}^{(2)}$ & 0.0198 & 0.0420 & 0.0974 & 0.9490 & 0.9814 & 0.9924 \\
\hline$n_{1}=n_{2}=50$ & $2.5 \%$ & $5 \%$ & $10 \%$ & $90 \%$ & $95 \%$ & $97.5 \%$ \\
\hline$\lambda_{1}^{(1)}=\lambda_{1}^{(2)}$ & 0.0249 & 0.0497 & 0.1027 & 0.9327 & 0.9706 & 0.9873 \\
\hline$n_{1}=n_{2}=100$ & $2.5 \%$ & $5 \%$ & $10 \%$ & $90 \%$ & $95 \%$ & $97.5 \%$ \\
\hline$\lambda_{1}^{(1)}=\lambda_{1}^{(2)}$ & 0.0241 & 0.0510 & 0.1037 & 0.9207 & 0.9634 & 0.9829 \\
\hline
\end{tabular}

Next, we consider the case of the contaminated normal distribution. We assume that $\boldsymbol{X}_{j}^{(i)}$ is distributed as follows

$$
0.7 \times N(\mathbf{0}, \operatorname{diag}(6,3,1))+0.3 \times N(\mathbf{0}, \operatorname{diag}(14,4,2)) .
$$

Under this assumption the variables $y_{j 1}^{(1)}, \cdots, y_{j N_{1}}^{(1)}, y_{j 1}^{(2)}, \cdots, y_{j N_{2}}^{(2)}$ are exchangeable. Table 4 shows the actual significance levels corresponding to the nominal levels under the contaminated normal distribution.

Finally, we examine the power of our test statistic based on permutation test. We assume that $\boldsymbol{\Sigma}^{(1)}=\operatorname{diag}(6,3,1)$ and $\boldsymbol{\Sigma}^{(2)}=\operatorname{diag}(8,3,1)$. For comparison we derived the exact distribution of the ratio of two largest characteristic roots in Section 3. In Table 5 we show the power of the numerical results based on the exact distribution and the power of the permutation test. 


\section{TAKEDA}

Table 4: Actual significance levels corresponding to the nominal levels for the contaminated multivariate normal distribution

\begin{tabular}{ccccccc}
\hline$n_{1}=n_{2}=20$ & $2.5 \%$ & $5 \%$ & $10 \%$ & $90 \%$ & $95 \%$ & $97.5 \%$ \\
\hline$\lambda_{1}^{(1)}=\lambda_{1}^{(2)}$ & 0.0235 & 0.0478 & 0.0995 & 0.9043 & 0.9555 & 0.9786 \\
$\lambda_{2}^{(1)}=\lambda_{2}^{(2)}$ & 0.0198 & 0.0423 & 0.0913 & 0.9124 & 0.9581 & 0.9808 \\
$\lambda_{3}^{(1)}=\lambda_{3}^{(2)}$ & 0.0356 & 0.0647 & 0.1157 & 0.8781 & 0.9349 & 0.9653 \\
\hline \hline$n_{1}=n_{2}=50$ & $2.5 \%$ & $5 \%$ & $10 \%$ & $90 \%$ & $95 \%$ & $97.5 \%$ \\
\hline$\lambda_{1}^{(1)}=\lambda_{1}^{(2)}$ & 0.0275 & 0.0526 & 0.1000 & 0.9046 & 0.9525 & 0.9764 \\
$\lambda_{2}^{(1)}=\lambda_{2}^{(2)}$ & 0.0209 & 0.0485 & 0.1028 & 0.9029 & 0.9515 & 0.9772 \\
$\lambda_{3}^{(1)}=\lambda_{3}^{(2)}$ & 0.0259 & 0.0555 & 0.1052 & 0.8967 & 0.9437 & 0.9709 \\
\hline \hline$n_{1}=n_{2}=100$ & $2.5 \%$ & $5 \%$ & $10 \%$ & $90 \%$ & $95 \%$ & $97.5 \%$ \\
\hline$\lambda_{1}^{(1)}=\lambda_{1}^{(2)}$ & 0.0243 & 0.0487 & 0.0993 & 0.8994 & 0.9474 & 0.9754 \\
$\lambda_{2}^{(1)}=\lambda_{2}^{(2)}$ & 0.0251 & 0.0495 & 0.0968 & 0.9024 & 0.9524 & 0.9749 \\
$\lambda_{3}^{(1)}=\lambda_{3}^{(2)}$ & 0.0273 & 0.0537 & 0.1061 & 0.8947 & 0.9452 & 0.9731 \\
\hline
\end{tabular}

Table 5: The power of permutation test and numerical result

\begin{tabular}{cccc}
\hline$n_{1}=n_{2}=20$ & $2.5 \%$ & $5 \%$ & $10 \%$ \\
\hline permutation & 0.063 & 0.113 & 0.215 \\
\hline numerical result & 0.096 & 0.160 & 0.261 \\
\hline & & & \\
\hline$n_{1}=n_{2}=50$ & $2.5 \%$ & $5 \%$ & $10 \%$ \\
\hline permutation & 0.147 & 0.239 & 0.373 \\
\hline numerical result & 0.173 & 0.263 & 0.392 \\
\hline & & & \\
\hline$n_{1}=n_{2}=100$ & $2.5 \%$ & $5 \%$ & $10 \%$ \\
\hline permutation & 0.283 & 0.399 & 0.541 \\
\hline numerical result & 0.297 & 0.414 & 0.557 \\
\hline
\end{tabular}

\section{Discussions}

In testing the equality of eigenvalues of two populations, we face the problem of the population distribution's being far from normality or of finding the true distribution to be very tedious. To resolve this problem, we have obtained the permutation test for equality of eigenvalues in two populations and obtained the exact distribution of the test statistic assuming normality.

We consider the reliability of the significance levels. In Table 1, we know that if the sample size is over 50 , the actual significance levels may be close to the nominal significance levels. In Table 2, the significance levels are the same as the Case 1. The test statistic $T$ depends on the sample characteristic vector $\boldsymbol{b}_{j}^{(i)}$. However the distribution of the test statistic $T$ depends on population covariance matrices $\boldsymbol{\Sigma}^{(1)}$ and $\boldsymbol{\Sigma}^{(2)}$, but does not depend 
on characteristic vectors $\boldsymbol{\beta}_{1}^{(i)}, \cdots, \boldsymbol{\beta}_{p}^{(i)}$ under the multivariate normal distribution. Thus the difference of the characteristic vectors has no influence. But Table 3 is not the same as the other cases. The expected value of $\ell_{j}^{(i)}$ is given by

$$
E\left[\ell_{j}^{(i)}\right]=\lambda_{j}^{(i)}+\frac{\lambda_{j}^{(i)}}{n_{i}} \sum_{k \neq j} \frac{\lambda_{k}^{(i)}}{\lambda_{j}^{(i)}-\lambda_{k}^{(i)}}+O\left(n_{i}^{-2}\right) .
$$

Hence $E\left[\ell_{1}^{(2)}\right]$ is larger than $E\left[\ell_{1}^{(1)}\right]$. This means that the statistic $T$ may be larger than 1 in Case 3. We may see that the actual significance levels are larger than the nominal levels in the upper tail. In Table 4, these results under the multivariate contaminated normal distribution are not inferior to those of the multivariate normal distribution. In general, the variable $\boldsymbol{b}_{j}^{(i)}$, the estimator of characteristic vector $\boldsymbol{\beta}_{j}^{(i)}$, depends on all characteristic roots $\lambda_{1}, \cdots, \lambda_{p}$. Thus the distribution of $z_{j k}^{(i)}$ depends weakly on $\lambda_{1}, \cdots, \lambda_{j-1}, \lambda_{j+1}, \cdots, \lambda_{p}$. If the characteristic roots are different in each population, it might require sufficiently large sample size, larger than 100 from Table 4, for our testing procedures to be reliable.

We consider the power of our permutation test. In Table 5 , the power of the permutation test is smaller than that of the numerical results. This is because in Table 1, 2 and 3 the significance levels of the simulation is conservative. As a result, the power of the permutation test is smaller. However each power in the largest characteristic roots and large sample size is not so different. Therefore we may expect similar results in other characteristic roots.

In many cases we note that the sample size 20 is too small to evaluate the performance, but for size over 100 we may say that the permutation test is guaranteed. In many applications, verifying the prior condition of normality and investigating the true population distribution is very difficult. Hence the roll of the permutation test is very important.

Besides the ratio of eigenvalues, we look for other possible powerful test statistics. Also we may know other non-parametric methods, such as a bootstrap method. Further work remains to be done in comparing the permutation method with the bootstrap method.

\section{Acknowledgements}

The author heartily thanks Professor Takakazu Sugiyama who gave him some useful advices and encouragements for working on this problem and read the original version of the manuscript. He also wishes to express very sincere thanks to referees and Professor Nariaki Sugiura for their valuable suggestions to improve the paper.

\section{REFERENCES}

Good, P. (1994). Permutation tests. New York: Springer-Verlag.

Konishi, S. (1977). Asymptotic expansion for the distribution of a function of latent roots of the covariance matrix. Annals of the Institute of Statistical Mathematics 29, Part A, 389-396.

Pesarin, F. (1999). Permutation testing of multidimensional hypotheses by nonparametric combination of dependent tests. Padova: Cleup Editrice.

Shiraishi, T. and Okuyama, S. (1991). Random Numbers. Journal of Yokohama City University (in Science) 43, 133-156.

Sugiura, N. (1973). Derivatives of the characteristic root of a symmetric or a Hermitian matrix with two applications in multivariate analysis. Communications in Statistics 1, 393-417. 


\section{TAKEDA}

Sugiyama, T. (1967). On the distribution of the largest latent root of the covariance matrix. Annals of Mathematical Statistics 38, 1148-1151.

Sugiyama, T. (1983). Introduction to multivariate data analysis. Tokyo: Asakura Shoten.

Sugiyama, T. and Ushizawa, K. (1998). A non-parametric method to test equality of intermediate latent roots of two populations in a principal component analysis. Journal of the Japan Statistical Society 28, 227-235.

(Received December 2000, Accepted November 2001) 